\title{
Percepción de los profesores sobre integración de TIC en las prácticas de enseñanza en relación a los marcos normativos para la profesión docente en Chile ${ }^{a}$
}

Marcelo Arancibia Herrerab

Daniela Cosimo Fernández ${ }^{b}$

Roberto Casanova Seguel ${ }^{b}$

\section{Resumen}

Investigación que busca relacionar las percepciones de profesores sobre el uso de tecnologías con la Política Pública "Estándares y Competencias TIC para la profesión docente". Estudio cualitativo en el que se aplican 40 entrevistas a profesores en 3 regiones de Chile. Los resultados evidencian una caracterización de los profesores según el uso que otorgaban a las tecnologías, la organización de matrices analíticas y el reconocimiento de las competencias TIC presentes en su discurso. Si bien la mayoría de profesores manifiestan tener competencias asociadas a la dimensión pedagógica, existe una amplitud de criterios y competencias reconocidas. Esta investigación es una primera aproximación al conocimiento e identificación de las competencias TIC que poseen los profesores en concordancia con la Política Pública definida a nivel País.

Palabras clave: competencias TIC. Política educativa. Formación de profesores. Percepciones profesores.

\section{Introducción}

El uso de las Tecnologías de la Información y Comunicación (TIC) ha trascendido todos los ámbitos de la vida, espacios y lugares, configurando una sociedad en la que es prácticamente impensable prescindir de ellas.

\footnotetext{
a Investigación Financiada por Conicyt, proyecto FONDECYT N¹1130316; patrocina Dirección de Investigación y Desarrollo Universidad Austral de Chile.

b Universidad Austral de Chile.
} 
En la actualidad, las nuevas generaciones demandan cambios en las prácticas pedagógicas convencionales. Para ello se requiere que los docentes desarrollen competencias vinculadas con estas nuevas necesidades; un ejemplo lo constituyen las denominadas competencias TIC, definidas como Política Pública en la mayoría de los países latinoamericanos.

La visión del trabajo que los profesores realizan con TIC está vinculada a la construcción de una nueva sociedad, que posibilite mayores oportunidades a personas en sus múltiples y complejas dimensiones: seres sociales, ciudadanos y trabajadores (BRASIL, 2011). Es en ese sentido que la presencia de las TIC aportaría mejoras a los establecimientos educacionales y, en general, al mundo de la educación.

En este contexto, resulta necesario conocer las percepciones de profesores en torno al uso educativo de las TIC: ¿cuáles son las competencias que creen poseer?; ¿cuán relacionadas están con el marco regulatorio ministerial?

\section{Escuela, profesorado y tecnología}

Los profesores son actores esenciales en el sistema educativo y en el logro de una buena enseñanza; su rol de guías, motivadores y facilitadores de recursos para diseñar el aprendizaje implica desplegar didácticas que incluyan materiales con diferentes soportes tecnológicos para facilitar el aprendizaje (CEBRIÁN, 2005). Asimismo, el docente debe asumir los cambios en forma crítica e innovadora para que los estudiantes: sean eje del proceso; exploren y elaboren nuevos conocimientos adquiriendo un rol activo en su formación; y generen competencias necesarias para integrarse al mundo digital con características sociales y culturales particulares (SALINAS, 2004).

Desde la utilización del computador con un fundamento motivacional en los años 80 , se ha transitado en la última década hacia la búsqueda del aprendizaje colaborativo (ARANCIBIA et al., 2014). Sin embargo, no siempre la evolución de las tecnologías, que ha devenido en procesos de convergencia digital, es acompañada de una transformación en términos de innovación pedagógica. Como afirma Castells (2001), las nuevas tecnologías no son solo herramientas que aplicar, sino procesos que desarrollar. Así, evidentemente el avance de la tecnología es más acelerado que el desarrollo de prácticas educativas que posibiliten una incorporación pedagógica de estos recursos en el aula, aspecto que implica procesos constantes de evaluación y revisión.

Para algunos autores, una de las aristas del problema es la manera en que se ha concretado la integración de tecnologías en las instituciones escolares. Gros, 
por ejemplo, afirma que este proceso se ha caracterizado por su carácter de "intrusión, como algo que necesariamente ha de utilizarse, sin saber muy bien por qué, para qué, cómo" (2000, p. 18). Frente a esto, propone una apropiación basada en necesidades pedagógicas, es decir, analizando aquellos aspectos que permitan un uso justificado y contextualizado en el entorno social en que las tecnologías se usarán. Esto resulta muy distinto a lo que se hace comúnmente: permitir que la tecnología se imponga a los usuarios y no que estos busquen una herramienta que satisfaga sus necesidades y traspase la barrera del uso meramente instrumental. Dicha idea también es compartida por Crook (1998), quien expone la problemática a partir de la metáfora del ordenador como tutor. Esta relación fundamenta la existencia de una percepción en el profesorado que conceptualiza al computador como un recurso tecnológico extraño - ajeno-, una intromisión en las prácticas docentes.

En una perspectiva más radical, Arancibia (2007) expone que una incorporación adecuada de la tecnología al interior de los centros educativos requiere de la reformulación de la actual organización escolar, la gestión pedagógica y el rol social de la escuela.

En diversos contextos se establecen parámetros y orientaciones que conllevan establecer políticas traducidas en estándares y competencias TIC para educadores (ALMERICH, 2011; RICOY et al., 2010); así, en Chile, se han definido también Competencias y Estándares TIC para la Profesión Docente (en adelante CE-TIC, CHILE, 2011).

En esta lógica, una implementación exitosa de tecnologías en las clases supone que el profesorado no solo debe comprender y aceptar su utilidad, ventajas y posibilidades para su quehacer, sino también definir marcos que dirijan la implementación de estas tecnologías para llevar a cabo objetivos educativos (SUÁREZ et al., 2013).

La rápida evolución y masificación de las TIC desde fines del siglo XX ha dado forma a la denominada Sociedad del Conocimiento o de la Información (UNESCO, 2013), que ha permeado la cotidianidad de la vida de las personas (CHIAPPE et al., 2013), razón por la que se ha demandado al sistema educacional una actualización de las prácticas y contenidos con el propósito de que estos sean coherentes con la nueva Sociedad.

Las TIC se han convertido en recursos fundamentales en los centros educacionales, y con ello la incorporación de estudiantes al mundo digital, 
constituyéndose en herramientas de valor y apoyo para el trabajo pedagógico (BECERRIL BALÍN; BADIA GARGANTÉ, 2013). Una de las demandas más importantes en este escenario dice relación con la responsabilidad frente a la disminución de desigualdades, las que no se producen solo por acceso a la tecnología, sino también por sus formas de utilización (ARANCIBIA; CARRASCO, 2006).

\section{TIC y políticas públicas}

Diversos gobiernos están formulando políticas con el fin de incorporar las TIC de manera integral en el currículo; estudios comparados demuestran que no ha sido fácil la adecuada instalación de las TIC en las escuelas de América Latina (BISSET ALVAREZ et al., 2015; LUGO et al., 2014). En consecuencia, en varios países se habla del acceso a tecnología como derecho básico asociado a políticas de inclusión, lo cual aún es un desafío no logrado (LUGO; BRITO, 2015; QUARTIERO et al., 2012). Mientras, este es un tema de discusión a nivel global debido a lo indispensable del uso de las tecnologías para participar activamente en la sociedad e integrarse al mundo actual (UNESCO, 2013).

En Chile, atendiendo al principio descrito anteriormente y al alero de una política pública sobre TIC con casi veinte años de trayectoria, en 2011 se aplicó por primera vez el SIMCETIC ${ }^{1}$, cuyos resultados constituyen una fuente de información acerca del desarrollo de habilidades TIC en estudiantes. Los resultados 2013 mostraron que solo el 1,8\% de los estudiantes se encuentra en nivel avanzado (estándar deseado); el 51,3\% alcanzó nivel intermedio; y el 46,9\% se ubicó en inicial. Otros resultados preocupantes indican que el porcentaje promedio es mayor mientras más alto es el grupo socioeconómico. Según estos datos, la diferenciación socioeconómica afecta el desarrollo de habilidades TIC de los estudiantes; por ello la implementación de tecnologías se encuentra muy lejos de cumplir el objetivo de avanzar hacia un país con más oportunidades.

Es un hecho que las TIC no son neutrales, la presencia de estas produce cambios positivos y negativos en el sistema educativo. Por esto, el impacto que produzcan en la escuela dependerá de cómo se utilicen, siendo el profesor uno de los mayores responsables en esta tarea (ERTMER; OTTENBREITLEFTWICH, 2010).

Sistema de Medición de la Calidad de la Educación (www.simce.cl). Sobre SIMCETIC véase http://www. enlaces.cl/evaluacion-de-habilidades-tic/simce-tic/presentacion/ 


\section{Análisis de competencias y estándares TIC para la profesión docente}

El Ministerio de Educación chileno (MINEDUC) publicó en 2011 las CE-TIC destinadas a la formación inicial docente (CHILE, 2011). Este documento define cinco dimensiones:

- Pedagógica (DP): integrar las TIC a los procesos de enseñanza y desarrollo de aprendizaje del estudiantado.

- Técnica (DT): incorporar las TIC con un equipamiento adecuado, aprendizaje de y con las tecnologías, entregando confianza en el uso de TIC.

- Gestión (DG): involucra las acciones administrativas realizadas y roles técnico/ pedagógicos en un establecimiento educacional con uso de TIC.

- Social, ética y legal (DE): labor de los docentes para que estudiantes se apropien de la implicancia del uso de TIC en un marco de respeto y compromiso, de cuidado de sí mismos, de los demás y del medio ambiente.

- Responsabilidad y desarrollo profesional (DR): uso de tecnologías para el perfeccionamiento docente, actividades de colaboración, consulta de fuentes de información, entre otras.

De cada dimensión surgen competencias definidas según criterios estandarizados. Consecuentemente, un estándar es una competencia que se vuelve un referente válido para un grupo. En síntesis, los estándares orientan respecto a cómo cumplir con lo que describen las competencias y criterios, explicitando logros de las competencias TIC.

- Para las cinco dimensiones anteriormente descritas, se definen cuatro competencias genéricas:

- Comunicación: escucha a otros, demuestra apertura para compartir información y conocimientos.

- Capacidad de planificar y organizar: plantea objetivos claros, asigna eficientemente el tiempo y recursos, monitorea las acciones emprendidas.

- Innovación: desarrolla opciones nuevas para mejorar los aprendizajes. 
- Compromiso con el aprendizaje continuo: busca desarrollarse a sí mismo en lo personal y profesional, contribuye al aprendizaje de sus colegas y de otros agentes de la comunidad.

La estructura descrita, diferenciada en cinco dimensiones y cuatro competencias, será el marco analítico del estudio.

\section{Metodología}

Esta investigación tiene como objetivo relacionar las percepciones de 40 profesores de Lenguaje, Matemática, Ciencias e Historia, sobre aprender y enseñar con TIC, con las CE-TIC propuestas por el MINEDUC, desde la "voz" de los protagonistas (ESCORCIA-OYOLA; JAIMES DE TRIVIÑO, 2015).

La investigación es cualitativa, lo cual otorga una característica de investigación holística; por ello es importante la observación de los datos e interpretación de la realidad de los casos de estudio, pues como manifiesta Pérez (2004) ello permite desarrollar categorías conceptuales, o ilustrar, defender o desafiar presupuestos teóricos. A continuación, la Tabla 1 resume las características de los cuarenta profesores entrevistados - lo que muestra su heterogeneidad - su distribución de acuerdo a género, edad, localización, años de ejercicio, lugar de trabajo y disciplina.

Nuestro estudio considera variables específicas para la selección de los informantes claves (STAKE, 1999): voluntad y disposición del profesor, uso de TIC en su labor docente y acceso a laboratorios de computación en sus respectivas instituciones.

El instrumento de recolección de información fue una entrevista semi-estructurada, aplicada el segundo semestre de 2015, y corresponde a una adaptación del instrumento empleado en una tesis doctoral ${ }^{2}$. La adaptación fue validada por tres jueces expertos de cada disciplina. Las transcripciones son analizadas en base a una matriz teórica que contempla dimensiones o bloques temáticos intencionados para que emerja el discurso de los profesores durante la entrevista, relacionados con los tópicos abordados en la investigación:

- (D1) Currículum: marco socio cultural, ideológico y político del sistema escolar; cuestiones del conocimiento de la disciplina. Considera conceptos sobre la elaboración del currículo en la escuela a través del rol de la planificación y de la implicación y sentido de la asignatura.

2 Es posible revisar el documento en: http://www.tdx.cat/handle/10803/126403 
Tabla 1. Resumen características profesores entrevistados.

\begin{tabular}{|c|c|c|c|c|c|}
\hline Género & Edad & Región & Años de ejercicio & Tipo de IE & Asignatura \\
\hline$M$ & 51 & Bío-Bío & 10 & PS & Ciencias \\
\hline M & 28 & & 3 & M & \\
\hline $\mathrm{F}$ & 35 & & 9 & PS & \\
\hline $\mathrm{F}$ & 44 & Los Ríos & 11 & PS & \\
\hline $\mathrm{F}$ & 44 & & 6 & PS & \\
\hline $\mathrm{F}$ & 26 & & 3 & M & \\
\hline $\mathrm{F}$ & 30 & & 4 & PS & \\
\hline $\mathrm{F}$ & 31 & Valparaíso & 3 & PP & \\
\hline $\mathrm{F}$ & 37 & & 13 & $M$ & \\
\hline $\mathrm{F}$ & 41 & & 14 & PS & \\
\hline M & 51 & Bío-Bío & 27 & PP & Historia \\
\hline M & 28 & & 2 & M & \\
\hline M & 27 & & 3 & $M$ & \\
\hline $\mathrm{F}$ & 35 & Los Ríos & 11 & PS & \\
\hline M & 38 & & 11 & PS & \\
\hline M & 56 & & 26 & PS & \\
\hline M & 31 & & 9 & $M$ & \\
\hline M & 47 & Valparaíso & 17 & PS & \\
\hline $\mathrm{F}$ & 39 & & 15 & $M$ & \\
\hline $\mathrm{F}$ & 43 & & 16 & PS & \\
\hline $\mathrm{F}$ & 25 & Bío-Bío & 2 & $M$ & Lenguaje \\
\hline $\mathrm{F}$ & 43 & & 20 & PS & \\
\hline M & 41 & & 13 & PP & \\
\hline $\mathrm{F}$ & 28 & Los Ríos & 6 & PS & \\
\hline $\mathrm{F}$ & 30 & & 4 & PS & \\
\hline M & 29 & & 6 & PS & \\
\hline M & 30 & & 7 & PS & \\
\hline $\mathrm{F}$ & 29 & & 7 & PS & \\
\hline M & 40 & Valparaíso & 22 & PS & \\
\hline $\mathrm{F}$ & 40 & & 17 & $M$ & \\
\hline M & 45 & Bío-Bío & 18 & PP & Matemáticas \\
\hline M & 48 & & 20 & PS & \\
\hline $\mathrm{F}$ & 27 & & 5 & PS & \\
\hline $\mathrm{F}$ & 33 & Los Ríos & 2 & PS & \\
\hline M & 42 & & 10 & $M$ & \\
\hline M & 47 & & 14 & PS & \\
\hline M & 56 & & 30 & PS & \\
\hline M & 30 & & 3 & M & \\
\hline $\mathrm{F}$ & 56 & Valparaíso & 33 & $M$ & \\
\hline M & 31 & & 4 & PS & \\
\hline
\end{tabular}

F: femenino; M: masculino; IE: institución escolar; M: municipal; PS: particular subvencionado; PP: particular pagado.

Fuente: elaboración propia (2015). 
- (D2) Acción didáctica: concepciones sobre aprendizaje, enseñanza y evaluación, entendiendo que en la práctica docente estas se interconectan en un proceso que tiene fases de preparación, acción y reflexión.

- (D3) Tecno/didáctica: concepciones sobre el efecto, relevancia y uso de las TIC. Indican la relación del profesor hacia las TIC y su efecto en la cultura escolar y en el proceso enseñanza aprendizaje.

La entrevista se estructuró en tres partes: la primera orientada a caracterizar el perfil y el contexto de los profesores; la segunda, constituida por veinte preguntas, indaga sobre currículum y didáctica; la tercera, con seis preguntas, caracteriza la dimensión tecno/didáctica. A continuación, en el Cuadro 1 presentamos ejemplos de preguntas:

Para el análisis del documento CE-TIC se elaboró un cuadro resumen según dimensiones, competencias, criterios y descriptores, a los cuales es asignado un código. Luego se hizo el cruce interpretativo entre estos códigos y el discurso de los profesores en las entrevistas, considerando la frecuencia de aparición de los códigos en las entrevistas.

\section{Resultados}

A continuación, se exponen los resultados del estudio; en primer lugar, la caracterización de las percepciones de los profesores sobre uso de TIC, y luego el contraste entre estas con las competencias y estándares TIC propuestos por el MINEDUC.

Cuadro 1. Ejemplos preguntas entrevistas.

\begin{tabular}{|l|c|}
\hline $\begin{array}{l}\text { Parte 1. } \\
\text { Perfil y contexto }\end{array}$ & $\begin{array}{c}\text { Año de obtención título: } \\
\text { Años de ejercicio docente: }\end{array}$ \\
¿Cómo se ha formado en el uso de TIC? \\
$\begin{array}{l}\text { Parte 2. } \\
\text { Currículum y } \\
\text { didáctica }\end{array}$ & $\begin{array}{r}\text { ¿Cuál es el sentido que asigna a la enseñanza de su disciplina en los } \\
\text { establecimientos educacionales? } \\
\text { ¿Cómo piensa que aprenden sus alumnos? }\end{array}$ \\
\hline $\begin{array}{l}\text { Parte 3. } \\
\text { Dimensión } \\
\text { tecnológica }\end{array}$ & $\begin{array}{r}\text { ¿Con qué rasgos identifica una buena práctica docente? } \\
\text { ¿Cómo utiliza los resultados de sus evaluaciones? }\end{array}$ \\
\hline
\end{tabular}

Fuente: elaboración propia (2017). 


\subsection{Caracterización de las percepciones de los profesores sobre el uso educativo de las TIC}

Primero se realiza una caracterización autoevaluada sobre uso y dominio de TIC para cada profesor. Para caracterizar el nivel de dominio de TIC de los profesores entrevistados, se dieron a conocer cuatro categorías: Básico, Intermedio, Avanzado y Experto (adaptadas de ALMERICH et al., 2011), con las que tendrían que identificarse. La descripción de las categorías es la siguiente:

- Básico: solo maneja internet y software de productividad de manera instrumental.

- Medio: elabora materiales con uso de software e internet de apoyo a su docencia.

- Avanzado: además de lo anterior, se comunica e interactúa por la red y sistemas informáticos con soltura.

- Experto: realiza todo lo anterior y además es capaz de enseñar a otros el uso de herramientas de productividad y apoyar a colegas en usos didácticos de TIC.

Como se observa en Tabla 2, dos profesores se identifican con un nivel de dominio básico de TIC (5\%). El 15\% de los profesores se considera en un nivel experto. Finalmente, la mayor cantidad de profesores se identifica con el nivel medio o avanzado $(80 \%)$.

En la Tabla 3 se indica el tipo de formación en TIC de los profesores. Se identifican tres tipos: inicial, permanente y aprendizaje autónomo (en esta pregunta se podía indicar más de una opción).

Tabla 2. Nivel de dominio de herramientas informáticas.

\begin{tabular}{lccccc}
\hline \multirow{2}{*}{ Conocimiento } & \multicolumn{5}{c}{ Área disciplinar } \\
\cline { 2 - 6 } & Lenguaje & Ciencias & Historia & Matemática & Total \\
\hline Básico & 0 & 1 & 0 & 1 & 2 \\
Medio & 4 & 4 & 6 & 5 & 19 \\
Avanzado & 4 & 4 & 3 & 2 & 13 \\
Experto & 2 & 1 & 1 & 2 & 6 \\
\hline
\end{tabular}

Fuente: elaboración propia (2016). 
Tabla 3. Descripción de formación del profesor en el uso de TIC.

\begin{tabular}{lccccc}
\hline \multirow{2}{*}{$\begin{array}{l}\text { Tipo de formación } \\
\text { en TIC }\end{array}$} & Lenguaje & Ciencias & Historia & Matemática & Total \\
\cline { 2 - 6 } & 8 & 4 & 4 & 6 & 22 \\
\hline $\begin{array}{l}\text { Formación inicial } \\
\text { universitaria }\end{array}$ & 3 & 2 & 0 & 4 & 9 \\
$\begin{array}{l}\text { Capacitación formación } \\
\text { permanente }\end{array}$ & 9 & 9 & 10 & 8 & 36 \\
Aprendizaje autónomo & 9 & & & & \\
\hline
\end{tabular}

Fuente: elaboración propia (2016).

Veintidós profesores declaran haber tenido formación universitaria inicial, solo 9 afirman haber desarrollado cursos de capacitación, 36 señalan que han tenido formación autónoma. Esto revela la autonomía de los profesores por mantenerse actualizados e informados con respecto al uso de las tecnologías, asociado a las pocas instancias formales de especialización.

También se aprecia que menos del 50\% de profesores señala haber aprendido a utilizar las TIC durante su formación inicial, dato que confirma la escasa enseñanza sobre TIC presente en las carreras de pedagogía en Chile (GARRIDO et al., 2013). Esto es preocupante ya que el uso educativo de TIC está influenciado por la experiencia vivida durante la formación inicial (RIASCOS et al., 2009). Tal como señala Graells (2001), la forma más efectiva para que los profesores apliquen las TIC en los procesos de enseñanza y aprendizaje es fomentar la utilización desde la universidad.

Una de las percepciones más recurrentes de los profesores de Lenguaje acerca del uso de TIC en el contexto escolar, es que estas son fuente importante para la realización de clases y preparación de material. Además, señalan la facilitación del aprendizaje gracias al uso de herramientas como videos, documentales, presentaciones power point que permiten desarrollar clases "lúdicas" y "entretenidas", manteniendo la concentración e interés de los alumnos debido a la cercanía y naturalidad con que los nativos digitales utilizan estas herramientas tecnológicas en su vida cotidiana.

Otro de los factores importantes es el empleo de las tecnologías como medio de comunicación entre los actores de la comunidad educativa: alumnos, profesores, apoderados y administrativos. Esta es una de las características de la "sociedad de información" donde el acceso al conocimiento, información y comunicación se realiza de forma instantánea en un tiempo inmediato (UNESCO, 2013). Asimismo, se menciona que los profesores tienen la responsabilidad de aprender sobre el uso de las tecnologías para poder utilizarlas con sus alumnos de forma eficiente, 
y de la misma forma poder guiarlos en el "buen uso" de estas, por ejemplo, en la búsqueda de información confiable, logrando la creación de productos para el desarrollo de sus capacidades y que no solo constituyan un elemento distractor para el desarrollo de la clase. Sin embargo, esto se contradice con la Tabla 2, donde se demuestra que solo 2 de los 10 profesores posee un dominio experto de las TIC.

Otro dato indicado por los profesores de Lenguaje es la falta de implementación de recursos tecnológicos en los establecimientos escolares, como data show, computadores y conexiones a internet. Sin embargo, destacan que a pesar de la existencia de algunos recursos, estos no son utilizados por falta de conocimiento y accesibilidad.

Los profesores de Ciencias tienden a ver las TIC como un apoyo hacia las tareas administrativas y pedagógicas: el uso de recursos tecnológicos sirve a la búsqueda de información, videos, películas, blogs y documentales, con la intención de facilitar el aprendizaje realizando clases más "dinámicas" y "lúdicas”; posibilitan que los estudiantes se motiven por aprender los contenidos de la asignatura. Además, los docentes destacan que debe existir un equilibrio en el uso de las tecnologías, pues un uso excesivo podría provocar desconcentración y desinterés por parte de los estudiantes.

Los profesores de Matemática generalizan en la utilidad para la búsqueda de información, además del uso de herramientas y software para la aplicación de los contenidos y conceptos aprendidos en la asignatura, mejorando la comprensión y motivación de los estudiantes. A pesar de esto, los docentes señalan que para ocupar las herramientas tecnológicas, aprender a usarlas, seleccionar información y software útiles para el aprendizaje de los estudiantes, se requiere de tiempo adicional a lo asignado para la planificación.

Otro elemento importante para los profesores de Matemática es la falta de conocimiento de los estudiantes para manejar herramientas y software educativos, a pesar del manejo y cercanía que estos tienen con la tecnología, redes sociales y aplicaciones de entretenimiento. Lo anterior reafirma datos recientes obtenidos por el SIMCE TIC que indican que el mayor porcentaje de jóvenes utiliza las tecnologías para actividades de entretención, mientras un número reducido de ellos lo hace para actividades asociadas al aprendizaje.

Las concepciones de los profesores de Historia se asemejan a las de los profesores de las demás asignaturas analizadas en aspectos como utilidad para la búsqueda de información, realización de tareas administrativas y pedagógicas, uso de herramientas 
tecnológicas para motivar y mejorar el aprendizaje de los estudiantes. Mencionan que una de las ventajas de utilizar TIC en las clases es que los alumnos tienen un alto manejo de estas; sin embargo, muchas veces constituyen un distractor. Por ello, una de las responsabilidades del profesor es manejar las tecnologías y mantenerse actualizado para poder orientar y guiar el aprendizaje de los estudiantes.

Este grupo de profesores menciona enseñar a sus estudiantes a utilizar software como Word, para utilizarlo en clases y en sus vidas cotidianas, confirmando así el consenso en torno a que el uso eficiente de las TIC es crucial para desenvolverse adecuadamente en la sociedad, la inserción en el mundo laboral, además de la posibilidad de conectarse con el mundo por medio internet.

Al comparar las caracterizaciones de los cuatro grupos resulta interesante destacar las similitudes con respecto al uso que otorgan los profesores a las tecnologías durante la enseñanza y aprendizaje de los estudiantes: búsqueda de información por parte de los estudiantes y profesores, complemento de contenidos, preparación de material y realización de trabajos. Por otro lado, reconocen que la presencia de las TIC afecta positivamente la comunicación con fines pedagógicos, tanto en estudiantes como en profesores, y entre los mismos estudiantes, fortaleciendo la interacción entre estos, hecho que potencia el trabajo grupal y el compañerismo.

Otra de las interesantes concepciones de los profesores en torno a las TIC es que a través de su utilización se puede mejorar la dinámica de clases, captar la atención de los estudiantes motivando la construcción de su aprendizaje. No obstante, es un aspecto limitante que muchos alumnos utilizan las tecnologías exclusivamente para actividades de entretención y no precisamente con fines educativos, por lo que algunos no manejan software o programas útiles en el desarrollo de las clases de estos sectores de aprendizaje, como mencionan en mayor medida los profesores de Matemática. Esto tiene relación con los datos obtenidos a partir del SIMCE TIC: solo el 3,3\% de los estudiantes maneja adecuadamente las tecnologías, mientras que el 46,2\% se ubica en un nivel inicial de manejo. Asimismo, los profesores consideran que una de las grandes falencias para el uso de las TIC es la falta de implementación y conectividad en los establecimientos educacionales; esto dificultaría la utilización de herramientas TIC en las aulas.

A modo de síntesis, podemos evidenciar que los profesores de las cuatro disciplinas tienen una concepción positiva acerca del uso de las tecnologías, destacando la importancia de estas en el desarrollo del aprendizaje de los estudiantes y facilitación en la realización de tareas pedagógicas y administrativas, con lo que potencian la optimización del tiempo, organización, búsqueda y almacenamiento de información para la planificación y elaboración de actividades pedagógicas. 
Otra de las distinciones significativas es que la mayoría de las disciplinas, con excepción del sector de Ciencias, cree que a pesar de que en los colegios existen las herramientas tecnológicas, estas no son utilizadas por falta de conocimiento o de tiempo. Es así como se destaca que los profesores deben tener la responsabilidad de manejar las TIC, actualizando sus conocimientos. Sin embargo, la falta de tiempo para el aprendizaje y especialización en el manejo de herramientas tecnológicas es un aspecto que afecta negativamente el uso e implementación de las TIC en procesos de enseñanza aprendizaje.

\subsection{Contraste entre percepciones de los profesores y competencias y estándares TIC}

A partir del análisis del documento CE-TIC se resumen las dimensiones con cada una de sus competencias, criterios y descriptores. Estas dimensiones fueron codificadas para su comprensión, cuyo resultado es el siguiente: Dimensión Pedagógica=DP, Dimensión Técnica=DT, Gestión=DG, Dimensión Social, ética y legal=DE, y Desarrollo y responsabilidad profesional $=\mathrm{DR}$. Asimismo, $\mathrm{C} 1, \mathrm{C} 2$ y C3 se refieren a las competencias asociadas a cada Dimensión, mientras c1, c2, c3, c4 y c5 señalan los Criterios de cada competencia. Finalmente, las letras a, b, c, d y e designan las diferentes descripciones para cada criterio asociado a una competencia. Además, utilizamos la codificación $\mathrm{f}(\mathrm{x})$ para indicar la frecuencia: ocasiones en que fueron encontrados los descriptores en el discurso de los profesores.

\section{a) Sector Lenguaje}

Se encontró la presencia de competencias TIC pertenecientes a las cinco dimensiones, entre las cuales la más frecuente fue la DP, que además contempló sus tres competencias. Esto se correlaciona con las caracterizaciones realizadas para los profesores de Lenguaje, en las que se destacan las actividades relacionadas con DP como la selección, adaptación e implementación de TIC en experiencias de aprendizaje de acuerdo al contexto y recursos disponibles, favoreciendo el dinamismo de la clase, mejorando la motivación y comprensión de los estudiantes. Igual situación ocurre con DG, que describe las TIC como facilitador de la labor docente, tanto administrativa como pedagógica, fomentando el uso de espacios virtuales para facilitar y promover la interacción con miembros de la comunidad educativa.

\section{b) Sector Ciencias}

Se encontraron competencias asociadas a las cinco dimensiones. DP es la dimensión que presenta mayor frecuencia, incluyendo sus tres competencias, al contrario 
de lo que ocurre con DE, mencionada una vez en las diez entrevistas analizadas. Asimismo, DP se sustenta con la caracterización realizada para los profesores de Ciencias: estos utilizan TIC en la planificación e implementación de ambientes para el desarrollo de experiencias de aprendizaje, por ejemplo en actividades relacionadas con la búsqueda y selección de información, además de la creación de experiencias que faciliten la comprensión de los estudiantes. La segunda dimensión con mayor frecuencia es DR, lo que coincide con la caracterización de los profesores de Ciencias, en la que se señala que estos utilizan TIC para la obtención de información, almacenamiento y organización de información.

\section{c) Sector Matemática}

En los profesores de Matemática existe una mayor frecuencia de las competencias asociadas a DP, vinculada con actividades como el desarrollo de experiencias con instrumentales que favorecen el aprendizaje de los estudiantes. Luego, y contrariamente a los demás sectores de aprendizaje, siguen en orden de frecuencia las competencias que integran DT. Esto coincide con los resultados de la caracterización de los usos de TIC, en donde se menciona que estos profesores utilizan TIC como recursos tecnológicos en los procesos de enseñanza a través de, por ejemplo, software matemático.

\section{d) Sector Historia}

Las competencias más frecuentes corresponden a DP, DG y DR. Esta última dimensión integra competencias como usar TIC en las actividades de formación continua y de desarrollo profesional, aplicar estrategias y procesos para la gestión de conocimiento mediado por TIC y reflexionar sobre los resultados del uso y manejo de TIC en el propio desarrollo profesional, diseñando e implementando acciones de mejora. Dentro de las caracterizaciones de los profesores de Historia aparece el sentido de una responsabilidad como profesores, que se centra no solo en enseñar contenidos, sino también en formar a los estudiantes cultural y socialmente mediante la conexión con el mundo.

Un análisis general sobre las competencias TIC que declaran los profesores de todos los sectores de aprendizaje se presenta la Tabla 4, que resume las competencias según sus dimensiones y frecuencias respectivas.

$\mathrm{Al}$ analizar las cuatro disciplinas se constata que la dimensión más frecuente es DP -integración de las TIC en los procesos de enseñanza y aprendizaje de los estudiantes - a partir de tres competencias que involucran, principalmente, 
Tabla 4. Resumen Competencias TIC y dimensiones

\begin{tabular}{|c|c|c|c|c|c|c|c|}
\hline Dimensión & Competencia & Lenguaje & Ciencias & Matemáticas & Historia & $\begin{array}{c}\text { Total } \\
\text { competenda }\end{array}$ & $\begin{array}{c}\text { Total } \\
\text { dimensión }\end{array}$ \\
\hline \multirow{3}{*}{ DP } & DP C1 & 16 & 29 & 30 & 24 & f88 & \multirow{3}{*}{ f194 } \\
\hline & DP C2 & 22 & 9 & 17 & 11 & f59 & \\
\hline & DP C3 & 12 & 7 & 9 & 8 & f36 & \\
\hline \multirow{2}{*}{ DG } & DG C1 & 20 & 14 & 12 & 13 & f59 & \multirow{2}{*}{$\mathrm{f} 70$} \\
\hline & DG C2 & 3 & 3 & 2 & 3 & $\mathrm{f} 11$ & \\
\hline \multirow{3}{*}{ DR } & DR C2 & 2 & 5 & 5 & 3 & $\mathrm{f} 15$ & \multirow{3}{*}{$\mathrm{f} 44$} \\
\hline & DR C3 & 3 & 4 & 5 & 6 & $\mathrm{f} 18$ & \\
\hline & DR C1 & 0 & 10 & 0 & 1 & $\mathrm{f} 11$ & \\
\hline \multirow{2}{*}{ DT } & DT C1 & 4 & 0 & 15 & 8 & $\mathrm{f} 27$ & \multirow{2}{*}{ f34 } \\
\hline & DT C2 & 1 & 3 & 2 & 1 & $\mathrm{f} 7$ & \\
\hline \multirow{2}{*}{$\mathrm{DE}$} & DE C1 & 1 & 0 & 4 & 1 & f6 & \multirow{2}{*}{ f8 } \\
\hline & DE C2 & 0 & 1 & 0 & 1 & $\mathrm{f} 2$ & \\
\hline
\end{tabular}

Fuente: elaboración propia (2016).

integrar TIC a la planificación de ambientes y experiencias de aprendizaje, integrar TIC en la implementación de experiencias de aprendizaje y generar sistemas de información y comunicación mediada por computadores.

Específicamente, la competencia más desarrollada por los profesores es integrar conscientemente TIC en sus planificaciones y actividades para mejorar los procesos de aprendizaje de sus estudiantes, según buenas prácticas o estrategias probadas anteriormente.

Asimismo, los profesores integran TIC en la implementación de ambientes y experiencias de aprendizaje (según recursos y contexto) para el desarrollo integral de los estudiantes. Es importante enfatizar que esta incorporación debe ser a través de propósitos y objetivos curriculares bien definidos, con la intención de que la integración de TIC posibilite la mejora de la calidad de los procesos de enseñanza y aprendizaje.

Otra dimensión con alta frecuencia es DG, que contempla prácticas que involucran el currículum: uso de TIC para mejorar y renovar procesos de gestión curricular, uso de TIC para tareas administrativas, controlando los tiempos de la labor docente y la programación del uso de TIC según actividades o tiempos requeridos. Así, según la caracterización de los profesores, las TIC son utilizadas tanto para tareas 
administrativas como pedagógicas, y son de gran ayuda para la eficacia de la labor docente, pues optimizan el orden de las planificaciones y reducen el tiempo dedicado a aquello; tiempo que muchas veces excede las horas destinadas para tareas docentes.

La tercera dimensión con mayor frecuencia es DR, cuyo propósito es que el profesor adquiera las competencias necesarias para cumplir con los objetivos de aprendizaje de sus estudiantes. Estas competencias pueden ser adquiridas durante toda la vida del docente por diferentes medios: perfeccionamientos, espacios de colaboración entre pares, búsqueda de información, etc., es decir, existe un compromiso por el aprendizaje continuo. DR contempla tres competencias esenciales: utilización de TIC para la formación continua y el mejoramiento de las prácticas docentes, aplicación de estrategias para la gestión de conocimiento mediado por TIC y, finalmente, reflexión sobre los resultados de uso y manejo de TIC. En el caso de las caracterizaciones de los profesores, podemos destacar que estos tienen consciencia de las acciones que deben realizar para el mejoramiento de su quehacer profesional.

Aunque DT es una dimensión implícita en las demás, es decir deviene de la necesidad de las demás competencias de utilizar recursos tecnológicos, digitales y espacios virtuales en los procesos de enseñanza y aprendizaje, esta no es una concepción explícita en el discurso de los profesores; sin embargo, mencionan que es relevante que los profesores sepan usar, buscar y seleccionar recursos digitales disponibles, como servicios de internet que posibilitan la comunicación de los estudiantes, tanto entre ellos como con el resto del mundo. Es el caso de los profesores de Historia, quienes sostienen que las TIC ayudarían a la conexión con el mundo y a la formación cultural de sus estudiantes. Además, se señala que se utilizan los recursos digitales para el apoyo de los procesos de enseñanza aprendizaje según sus funcionalidades y objetivos de enseñanza planteados: uso de power point, youtube, blog, data show, computadoras y otros, que contribuyen a la realización de clases más cercanas a las experiencias e intereses de los "nativos digitales".

Otro resultado relevante es la baja frecuencia de una de las dimensiones más importantes para el desarrollo de la educación y que explicita aquello que un profesor debiera llevar a cabo: la Dimensión social, ética y legal, que contempla la incorporación de TIC en un marco de respeto y compromiso con el cuidado de sí mismo, de los demás y del medio ambiente (CHILE, 2011). Constituyen esta dimensión las competencias: la integración de TIC para el desarrollo de habilidades sociales, fomentando que los estudiantes participen de comunidades virtuales y desarrollando la comunicación, participación y aprendizaje colaborativo; y la incorporación de TIC a prácticas que favorezcan el respeto a la diversidad, igualdad de trato y condiciones saludables en el acceso y uso de estas. Esta última es de 
suma importancia, según algunos autores, para los propósitos de la educación en la actualidad: formar personas y ciudadanos críticos, conscientes y responsables con su entorno, además de generar las competencias necesarias para las características sociales y culturales este siglo (SALINAS, 2004), de manera que las tecnologías aporten a los procesos sociales, culturales y políticos (UNESCO, 2013).

Una diferencia encontrada en el análisis de dimensiones y competencias es que en Lenguaje existe un leve aumento en la frecuencia de la DG, lo que tendría relación con la labor relacionada a la asignatura y concepciones de los profesores en relación a la utilidad de las TIC: los profesores de Lenguaje manifiestan la concepción de que son ellos quienes tienen la responsabilidad de saber utilizar e integrar las tecnologías en las clases, aspecto que presenta relación con las competencias integradas en DG, señalando que se deben usar las TIC para mejorar y renovar procesos de gestión curricular, cuyos criterios son el análisis e interpretación del desempeño académico y de eficiencia interna, relacionado con las acciones de mejoramiento.

En el sector de Ciencias, la DT es levemente más frecuente que en Lenguaje y comunicación. Esta dimensión contempla competencias como usar recursos tecnológicos y digitales y operar sistemas digitales de información y comunicación en los procesos de enseñanza y aprendizaje de los estudiantes. Esto se relaciona con las caracterizaciones de los profesores de Ciencias, quienes señalan la utilización de herramientas tecnológicas para realizar las presentaciones de sus clases.

\section{Conclusiones}

Para desenvolverse en la actual sociedad de la información y del conocimiento, es crucial manejar competencias y habilidades tecnológicas. Para su desarrollo, la escuela y el profesor tienen un rol fundamental, ya que el impacto que produzca el uso de TIC dependerá de la forma en que el profesor las utilice para facilitar y guiar el aprendizaje de sus estudiantes. El grupo de profesores del estudio evidenció un alto nivel en el manejo de TIC (avanzado o medio), sin embargo, llama la atención que la mayoría declara haberse formado de manera autónoma, pese a la gran cantidad de oferta de cursos gratuitos del MINEDUC; ello hace suponer que no hay necesariamente una relación entre su aprendizaje de las TIC y las demandas o exigencias desde la normativa ministerial.

El uso de las tecnologías tiene relación directa con las percepciones de los profesores acerca de estas, y también sobre las experiencias que han tenido: si el profesor observa logros de aprendizajes en sus estudiantes con ayuda de TIC, es probable que siga utilizando estas herramientas; de lo contrario, es probable que las abandone. 
Es interesante que para los profesores de Matemáticas el uso de TIC se perciba más como una dificultad que una ayuda, mientras que para Lenguaje, Ciencias e Historia se percibe una utilidad centrada en las características lúdicas de las herramientas tecnológicas. Se observa en las cuatro disciplinas un uso centrado en la búsqueda de información, y se acusa falta de tiempo y saberes tecnológicos en docentes y estudiantes para ampliar su uso.

A pesar de que gran parte de profesores manifiesta tener mayormente competencias asociadas a la Dimensión Pedagógica, no existe un discurso homogéneo, es decir, existe amplitud de criterios y competencias reconocidas. Además, hubo otras competencias que no se expresaron, a pesar de su importancia para la realización de las tareas docentes; sin embargo, esto no quiere decir que los profesores no las desarrollen, sino que simplemente ellas no se manifestaron en su discurso. De hecho, la Dimensión Ética del uso de TIC es prácticamente inexistente en el discurso de los profesores de la muestra.

Con todo, las percepciones de los profesores de la muestra están en relación con las competencias TIC que manifiesta el Ministerio de Educación en Chile; sin embargo, esta coherencia es relativa, dada la ausencia en el discurso de los profesores de algunas competencias declaradas, en especial la casi ausencia de la dimensión ética y la excesiva focalización en la dimensión pedagógica, relegando las dimensiones de gestión, responsabilidades profesionales y técnicas a menores vinculaciones entre el discurso y la propuesta normativa.

Urge repensar en qué medida los documentos de política pública se traspasan a la acción didáctica en el nivel meso y micro curricular, pues los resultados de este estudio indican que el profesorado centra su atención solo en lo concerniente a lo propiamente pedagógico de las TIC, en el entendido de que el uso educativo de TIC es un componente más amplio y de mayor impacto en el aprendizaje escolar, tal cual evidencia el documento de CE-TIC. Es, por tanto, un desafío a la formación inicial y continua de profesores desarrollar estrategias que permitan comprender el uso pedagógico de TIC desde matrices didácticas más complejas que incluyan al menos las cinco dimensiones expuestas en el documento.

Esta investigación es una primera aproximación al conocimiento e identificación de las competencias TIC que poseen los profesores a partir de sus propias concepciones $\mathrm{y}$, al mismo tiempo, un acercamiento para descubrir cómo ocurre la formación de futuros habitantes de una sociedad cada vez más digitalizada. Con todo, al ser un desafío planteado de manera global a los sistemas educativos regionales y locales, este estudio propone la necesidad de seguir analizando las concepciones docentes respecto del uso de TIC y su relación con lo que demandan las políticas públicas en función de hacer cada vez más coherente la bajada al aula de dichos instrumentos normativos. 


\title{
Percepção dos professores sobre a integração das TIC nas práticas de ensino sobre marcos regulatórios para a profissão docente no Chile
}

\section{Resumo}

Pesquisa que busca relacionar as Percepção dos professores sobre o uso da tecnologia e sua relação com a política pública de "Normas e competências TIC para a Profissão Docente”. Estudo qualitativo de 40 entrevistas com professores em 3 regiões do Chile. Os resultados mostram uma caracterização dos professores, dependendo do uso concedido à tecnologia, a organização de rubricas e reconhecimento de competências TIC no discurso dos professores. Ainda que a maioria dos professores dizem que têm habilidades associadas à dimensão educativa, há uma vasta serie de critérios e competências reconhecidas. Esta pesquisa é uma abordagem inicial do conhecimento e a identificação de competências em TIC nos professores de acordo com a politica pública do Chile.

Palavras-chave: Competências em TIC. Politica de educação. Formação de professores. Percepção dos professores.

\section{Teachers' perception about ICT integration in teaching practices in relation to the normative frameworks for the teaching profession in Chile}

\begin{abstract}
This research aims to relate teachers' perceptions to the use of technologies, and their relationship with the public policy "Standards and ICT competencies for the teaching profession". It is a qualitative study with 40 teachers, from three Chilean regions. An interview was applied. The main results show a characterization of teachers depending on the use granted to technology, organization of rubrics and finally, recognition of ICT skills in their discourse. While most of teachers say they have skills associated with the educational dimension, there is no a homogeneous discourse in the teachers who participated in the study, that is to say, there is a wide range of criteria and recognized skills. This research is a first approach to knowledge and identification of ICT skills that teachers have in accordance with the Public Policy defined in the Country.
\end{abstract}

Keywords: ICT competence. Educational policy. Teacher training. Teachers 'perception. 


\section{Referencias}

ALMERICH. G., et al. Las competencias y el uso de las Tecnologías de la Información y Comunicación por el profesorado: estructura dimensional. Revista Electrónica de Investigación Educativa, v. 13, n. 1, p. 28-42, 2011.

ARANCIBIA, M. Uso de medios y TIC en escuelas. ¿Cómo disminuir la brecha digital? Visiones de la Educación, n. 12, p. 17-30, 2007.

ARANCIBIA, M.; CARRASCO, Y. Incorporación de computadores en escuelas rurales. Estudio descriptivo de cuatro casos del sur de Chile. Estudios Pedagógicos, v. 32, n. 2, p. 7-26, 2006.

ARANCIBIA, M.; OLIVA, I.; PAIVA, F. Procesos de significación mediados por una plataforma de aprendizaje colaborativo desde los protagonistas. Comunicar, v. 21, n. 42, p. 75-85, 2014. https://doi.org/10.3916/C42-2014-07

BECERRIL BALÍN, L.; BADIA GARGANTÉ, A. La competencia informacional en la educación secundaria: demanda de aprendizaje y resolución colaborativa de problemas relativos a la información con apoyo de las TIC. Revista de Educación, n. 362, p. 659-89, 2013. https://doi.org/10.4438/1988-592X-RE-2013-362-245

BISSET ALVAREZ, E.; CARVALHO, A. M. G.; VIDOTTI, S. A. B. G. Políticas públicas de inclusión digital: el caso de América Latina y Cuba. Biblios, n. 58, p. 42-53, 2015. https://doi.org/10.5195/biblios.2015.203

CASTELLS, M. La era de la información: economía, sociedad y cultura: la sociedad de red. Madrid: Alianza Editorial, 2001. Vol. 1.

CEBRIÁN, M. (Coord.). Tecnologías de la información y comunicación para la formación de docentes. Madrid: Pirámide, 2005.

CHIAPPE, A.; MESA, N.; ÁLVAREZ, C. Transformaciones en las Concepciones de los Docentes de Educación Secundaria acerca de la Web 2.0 y su uso en los procesos de enseñanza. Estudios Pedagógicos (Valdivia), v. 39, n. 2, p. 55-66, 2013. https://doi.org/10.4067/S0718-07052013000200004

CHILE. Ministerio de la Educacion. Competencias y estándares TIC para la profesión docente. Santiago de Chile, 2011.

. Desarrollo de habilidades digitales para el siglo XXI en Chile ¿qué dice el SIMCE TIC? Santiago de Chile, 2013. 
CROOK, C. Ordenadores y aprendizaje colaborativo. Madrid: Ediciones Morata. 1998.

ESCORCIA-OYOLA, L.; JAIMES DE TRIVIÑO, C. Tendencias de uso de las TIC en el contexto escolar a partir de las experiencias de los docentes. Educación y Educadores, v. 18, n. 1, p. 137-52, 2015. https://doi.org/10.5294/edu.2015.18.1.8

ERTMER, P.; OTTENBREIT-LEFTWICH, A. Teacher technology change: how knowledge, confidence, beliefs, and culture. Intersect Journal of Research on Technology in Education, v. 42, n. 3, p. 255-84, 2010.

GARRIDO, J.; CONTRERAS, D.; MIRANDA, C. Análisis de la disposición pedagógica de los futuros profesores para usar las TIC. Estudios Pedagógicos, v. 39, n. especial, p. 59-74, 2013. https://doi.org/10.4067/S0718-07052013000300005

GRAELLS, P. Algunas notas sobre el impacto de las TIC en la universidad. Educar, v. 28, p. 83-98. 2001. https://doi.org/10.5565/rev/educar.391

GROS, B. El ordenador invisible: hacia la apropiación del ordenador en la enseñanza. Barcelona: Gedisa, 2000.

LUGO, M.T.; LÓPEZ, N.; TORANZOS, L. Politicas TIC en los Sistemas educativos de América Latina. Buenos Aires: Siteal/Unesco, 2014.

LUGO, M. T.; BRITO, A. Las políticas TIC en la educación de América Latina: una oportunidad para saldar deudas pendientes. Archivos de Ciencias de la Educación, v. 9, n. 9, p. 1-16, 2015.

PÉREZ, G. Investigación cualitativa: retos e interrogantes: métodos. Madrid: La Muralla, 2004.

QUARTIERO, E.; BONILLA, M.; FANTIN, M. Políticas para la inclusión de las TIC en las escuelas públicas brasileñas: contexto y programas. Campus Virtuales, v. 1. n. 1. p. 115-26, 2012.

RIASCOS, S.; QUINTERO, D.; ÁVILA, P. Las TIC en el aula: percepciones de los profesores universitarios. Educación y Educadores, v. 12, n. 3, p. 133-57, 2009.

RICOY, M.; FELIZ, T.; SEVILLANO, M. Competencias para la utilización de las herramientas digitales en la sociedad de la información. Educación, v. 13, n. 1, p. 199-219, 2010.

SALINAS, J. Innovación docente y uso de las TIC en la enseñanza universitaria. Revista de Universidad y Sociedad del Conocimiento, v. 1, n. 1, p. 1-16, 2004. 
SUÁREZ, J. et al. Las competencias del profesorado en TIC: estructura básica. Educación, v. 16, n. 1, p. 39-62, 2013. https://doi.org/10.5944/educxx1.16.1.716

STAKE, R. Investigación con estudios de casos. Madrid: Morata, 1999.

UNITED NATIONS EDUCATIONAL SCIENTIFIC AND CULTURAL ORGANIZATION - UNESCO. Enfoques estratégicos sobre las TIC en educación en América latina y el Caribe. Santiago: Oficina Regional de Educación para América Latina y el Caribe, 2013.

\section{Informacion de los autores}

Marcelo Arancibia Herrera: Profesor de Historia y Geografía, magister en educación y doctor en Sociedad de la Información y el Conocimiento. Universidad Austral de Chile. Contato: marceloarancibia@uach.cl

Daniela Cosimo Fernández: Profesora Enseñanza Media en Biología. Universidad Austral de Chile.cosimo.daniela@gmail.com

Roberto Casanova Seguel: Profesor de Lenguaje y Comunicación, magister en educación. Universidad Austral de Chile. Contato: roberto.casanova@uach.cl 